\title{
Forecasts on the Dark Energy and Primordial Non-Gaussianity Observations with the Tianlai Cylinder Array
}

\author{
Yidong $\mathrm{Xu}^{1}$, Xin Wang ${ }^{2}$, Xuelei Chen ${ }^{1,3}$
}

\begin{abstract}
The Tianlai experiment is dedicated to the observation of large scale structures (LSS) by the $21 \mathrm{~cm}$ intensity mapping technique. In this paper we make forecasts on its capability at observing or constraining the dark energy parameters and the primordial non-Gaussianity. From the LSS data one can use the baryon acoustic oscillation (BAO) and the growth rate derived from the redshift space distortion (RSD) to measure the dark energy density and equation of state. The primordial non-Gaussianity can be constrained either by looking for scale-dependent bias in the power spectrum, or by using the bispectrum. Here we consider three cases: the Tianlai cylinder array pathfinder which is currently being built, an upgrade of the pathfinder array with more receiver units, and the full-scale Tianlai cylinder array. Using the full-scale Tianlai experiment, we expect $\sigma_{w_{0}} \sim 0.082$ and $\sigma_{w_{a}} \sim 0.21$ from the BAO and RSD measurements, $\sigma_{\mathrm{f}_{\mathrm{NL}}}^{\text {local }} \sim$ 14 from the power spectrum measurements with scale-dependent bias, and $\sigma_{\mathrm{f}_{\mathrm{NL}}}^{\text {local }} \sim 22$ and $\sigma_{\mathrm{f}_{\mathrm{NL}}}^{\text {equil }} \sim 157$ from the bispectrum measurements.
\end{abstract}

Subject headings: cosmological parameters - large-scale structure of universe

\section{Introduction}

Baryon acoustic oscillations (BAO) are the frozen sound waves which were present in the photon-baryon plasma prior to the recombination epoch, and they imprint features on the cosmic microware background (CMB) as well as the large scale structures in the later Universe. The (comoving) characteristic scale of the BAO is determined by the sound horizon at the last scattering surface:

$$
s=\int_{0}^{t_{\mathrm{rec}}} c_{s}(1+z) d t=\int_{z_{\mathrm{rec}}}^{\infty} \frac{c_{s} d z}{H(z)}
$$

where $c_{s}$ is the sound speed, $H(z)$ is the Hubble expansion rate, and $t_{\text {rec }}$ and $z_{\text {rec }}$ are the recombination time and redshift respectively. The BAO scale provides a standard ruler to measure

\footnotetext{
${ }^{1}$ National Astronomical Observatories, Chinese Academy of Sciences, Beijing 100012, China

${ }^{2}$ Department of Physics \& Astronomy, Johns Hopkins University, Baltimore, MD, US, 21218

${ }^{3}$ Center for High Energy Physics, Peking University, Beijing 100871, China
} 
the angular diameter distance $D_{A}(z)$ and the Hubble parameter $H(z)$ Seo \& Eisenstein 2003; Blake \& Glazebrook 2003), hence serves as a promising tool to constrain the properties of dark energy, which determines the expansion rate of the Universe. This technique has successfully been used to put cosmological constraints on dark energy parameters from optical surveys (e.g. Anderson et al. 2014).

In addition to observing the cosmic expansion history through the BAO features in the matter power spectrum, a large scale structure measurement can also yield the structure growth rate $f(z)$ from redshift space distortions (RSD). The RSD features in the galaxy distribution have also been used to constrain the cosmological parameters, and to distinguish dark energy and various modified gravity models (e.g. Guzzo et al. 2008; Wang 2008; Linder 2008; Beutler et al. 2014; Samushia et al. 2014). The BAO and RSD features complement each other, and may also help break the degeneracy in dark energy and modified gravity models.

The large scale structures in the Universe could also be measured through the $21 \mathrm{~cm}$ emission from neutral hydrogen (HI) in galaxies, though current radio observations of $\mathrm{HI}$ in galaxies, e.g. the ALFALFA survey (Giovanelli et al. 2005) have been limited to redshift $z \lesssim 0.2$ at present. While some telescopes which are currently being constructed, e.g. the FAST (Nan et al. 2000), ASKAP (Johnston et al. 2008; Booth et al. 2009), and MeerKAT(Nan et al. 2000), will have much greater sensitivities, HI survey of galaxies at high redshift would still be a challenging task. Even for the Square Kilometer Array (SKA), with its wide area HI galaxy survey, the constraining power on cosmological parameters is only comparable to the existing optical galaxy surveys (Rawlings et al. 2004; Abdalla et al. 2010).

However, power spectrum constraints on cosmological paramters can be efficiently obtained with dedicated telescopes with lower resolution than galaxy surveys. Instead, one could observe in the intensity mapping mode, in which each pixel or voxel contains many galaxies. Many epoch of reionization experiments are in fact intensity mapping experiments, and the same method can also be used to observe large scale structure at the redshift range $0<z<3$, which is predicted to be more sensitive to cosmological parameters than current galaxy surveys (Chang et al. 2008; Peterson et al. 2009). This method is tested with existing telescopes such as the Green Bank Telescope (GBT) and the Parkes telescope, and has detected positive cross-correlation between the $21 \mathrm{~cm}$ and optical galaxies (Chang et al. 2010; Masui et al. 2013; Switzer et al. 2013). However, the time available on these general purpose telescopes are limited. It has been argued that large cylinderical reflectors can be cheaply made and used in hydrogen survey experiments (Peterson et al. 2006).

In a cylinder array designed for this purpose, a number of cylinder reflectors are fixed on the ground pointing the zenith, along the north-south direction and in parallel with each other. Receiver feeds are placed along the focus line of each of the cylinders, forming an interferometer array. As the Earth rotates, the array will drift scan the visible sky. For the observation of the $\mathrm{BAO}$ features in the large scale structure, which can be used to probe dark energy properties, a cylinder array of 100m-150m size would be optimal (Ansari et al. 2008, 2012; Seo et al. 2010). 
The Tianlai project 1 is an experimental effort in this direction (Chen 2012, 2011). Tentatively, we shall assume in this paper that the full-scale Tianlai experiment will consist of 8 adjacent cylinders, each with $15 \mathrm{~m}$ wide and $120 \mathrm{~m}$ long, totally with about 2000 dual polarization units, covering the frequency range of $400-1420 \mathrm{MHz}$, corresponding to $0<z<2.5$. We shall assume a system temperature of $50 \mathrm{~K}$.

At present, a pathfinder experiment is being built in a radio quiet site at Hongliuxia, Balikun County, Xinjiang Autonomous Region, China. This pathfinder consists of both a cylinder array and a dish array. The dish array will include 16 steerable $6 \mathrm{~m}$ dishes, and it will be discussed in a separate paper, here we shall focus on the cylinder array. The cylinder pathfinder array include three adjacent cylindrical reflectors, each with $15 \mathrm{~m}$ wide and $40 \mathrm{~m}$ long. It will focus on observing at the frequency range of $700-800 \mathrm{MHz}$. Currently, the pathfinder cylinders have a total of 96 receivers, averaging 32 on each. With a margin of 5 meters on each of the two ends of the cylinder, the distance between the feeds is around $97 \mathrm{~cm}$, greater than one wavelength at $z=1\left(\lambda_{\mathrm{obs}}=21(1+z) \mathrm{cm}\right)$. After a period of experiment, we plan to expand the total number of dual polarization receivers later to 216, so that on each cylinder there are on average 72 dual polarization receivers. We shall call this the pathfinder+ experiment. Using the pathfinder and pathfinder+ experiment, we hope to demonstrate the feasibility of intensity mapping with the cylinder array, before building the full-scale experiment. These configuration parameters for the cylinder pathfinder, pathfinder+ and full-scale experiment are listed in Table 1.

Another potentially interesting application of a $21 \mathrm{~cm}$ intensity mapping experiment is to look for and constrain the primordial non-Gaussianity. The primordial density perturbations, which were originated during the inflation era and gave rise to the various structures today, link the observable Universe to the very early phase of the Universe. While the simplest slow-roll inflation model predicts very weak primordial non-Gaussianity in the density perturbations with an amplitude below the detectable level, many other inflation mechanisms could result in observable non-Gaussianities (see Bartolo et al. 2004; Chen 2010 for reviews). Any observational constraint on the level of the primordial non-Gaussianity can be a powerful probe to the dynamics of inflation.

Various observational approaches, such as the angular bispectrum of the CMB, high-order correlations of the three dimensional galaxy distribution, abundance of rare objects, and the large-scale clustering of halos, have been developed to constrain the level of primordial non-Gaussianity, specifically the nonlinearity parameter $f_{\mathrm{NL}}$ (see Liguori et al. 2010; Verde 2010 for reviews). Combining large scale clustering measurements from galaxy surveys with their cross-correlations with the CMB from the Wilkinson Microwave Anisotropy Probe (WMAP) nine-year data, Giannantonio et al. (2014) obtained $-37<f_{\mathrm{NL}}<25$ at $95 \%$ confidence for the local-type configuration. The latest and tightest constraints on $f_{\mathrm{NL}}$ come from the measurement of the CMB angular bispectrum by Planck, which are $f_{\mathrm{NL}}^{\text {local }}=2.7 \pm 5.8, f_{\mathrm{NL}}^{\text {equil }}=-42 \pm 75$, and $f_{\mathrm{NL}}^{\text {ortho }}=-25 \pm 39(68 \% \mathrm{CL})$ for the primordial

${ }^{1}$ http://tianlai.bao.ac.cn. The word Tianlai means "heavenly sound" in Chinese, this phrase appeared first in the work of ancient Chinese philosopher Chuang Tzu (369BC-286BC). 
local, equilateral, and orthogonal bispectrum amplitudes respectively (Planck Collaboration et al. 2013b). Using the large scale clustering of tracers of dark matter in the later Universe, two most commonly used probes for the primordial non-Gaussianity are the scale-dependent bias in the observed power spectrum, and the bispectrum.

In this paper we make simple forecasts on the constraining power of the Tianlai experiment, under the assumption of perfect foreground removal and no systematics. We shall make our forecasts primarily for the full-scale experiment, which is designed to measure the large scale structure and cosmological parameters. We will also make some forecasts on the pathfinder and pathfinder+ experiments, which are only used for testing the key technology for the full-scale experiment and are not expected to achieve any good precision.

The paper is organized as follows: in Sec.2, we present the signal power spectrum, as well as the detailed formalism for estimating the noise power spectrum for an interferometer array, and forecast the measurement error of the power spectrum by the Tianlai arrays. Based on the power spectrum measurement, we forecast the constraints on dark energy parameters obtainable from Tianlai BAO and RSD observations in Sec.3. In Sec.4, we briefly review the imprint of primordial non-Gaussianity on the large scale structures, in Sec.4.1 we study the constraint obtainable by considering the scale-dependent bias in the power spectrum, and in Sec.4.2 we apply the bispectrum method. We conclude in Sec.5.

Table 1: The experiment parameters for Tianlai.

\begin{tabular}{|c|c|c|c|c|c|}
\hline & cylinders & width & length & dual pol. units/cylinder & Frequency \\
\hline Pathfinder & 3 & $15 \mathrm{~m}$ & $40 \mathrm{~m}$ & 32 & $700-800 \mathrm{MHz}$ \\
\hline Pathfinder + & 3 & $15 \mathrm{~m}$ & $40 \mathrm{~m}$ & 72 & $700-800 \mathrm{MHz}$ \\
\hline Full scale & 8 & $15 \mathrm{~m}$ & $120 \mathrm{~m}$ & 256 & $400-1420 \mathrm{MHz}$ \\
\hline
\end{tabular}

\section{The Power Spectrum Measurement with Tianlai Intensity Mapping}

\subsection{The Signal Power Spectrum}

In an HI intensity mapping observation, the distances along and perpendicular to the line of sight are measured from redshift and angular separation respectively, and the HI power spectrum is observed in redshift space. Therefore, the observed HI power spectrum is given by (Seo \& Eisenstein 2003)

$$
P_{\mathrm{obs}}\left(k_{\mathrm{ref} \perp}, k_{\mathrm{ref} \|}\right)=\frac{D_{A}(z)_{\mathrm{ref}}^{2} H(z)}{D_{A}(z)^{2} H_{\mathrm{ref}}(z)}\left(b_{1}^{\mathrm{HI}}(z)+f(z) \frac{k_{\|}^{2}}{k_{\perp}^{2}+k_{\|}^{2}}\right)^{2} \times G(z)^{2} P_{\mathrm{m} 0}(k)+P_{\mathrm{shot}},
$$


where the subscript "ref" denotes quantities calculated in the reference cosmology, for which we use the Planck 2013 parameters (Planck Collaboration et al. 2013a). Here $b_{1}^{\mathrm{HI}}(z)$ is the linear bias factor of the HI gas at redshift $z, f(z)$ is the linear growth rate, $k_{\perp}$ and $k_{\|}$are the $\mathbf{k}$ components perpendicular and parallel to the line of sight, respectively, $G(z)$ is the growth factor, $P_{\mathrm{m} 0}(k)$ is the present matter power spectrum, and $P_{\text {shot }}$ is the shot noise contribution.

In a model with dark energy equation of state $w(z)$, the hubble parameter and the angular diameter distance are given by

$$
\frac{H(z)}{H_{0}}=\left[\Omega_{m}(1+z)^{3}+\Omega_{k}(1+z)^{2}+\Omega_{X} e^{3 \int_{0}^{z} \frac{1+w\left(z^{\prime}\right)}{1+z^{\prime}} d z^{\prime}}\right]^{1 / 2}
$$

and

$$
D_{A}(z)=\frac{c}{1+z} \int_{0}^{z} \frac{d z^{\prime}}{H\left(z^{\prime}\right)} .
$$

Thus the present density and the equation of state parameters of dark energy can be constrained by measuring the acoustic peaks on the power spectrum.

The redshift space distortion of the power spectrum also provides information on the growth history of the Universe. The linear growth rate $f(z)$ affects the observed power spectrum (Eq. (2)) through the redshift space distortion (RSD) factor $\beta$, by

$$
\beta=f(z) / b_{1}^{\mathrm{HI}}(z)
$$

and through the linear growth factor $G(z)$, which is related to $f(z)$ by

$$
f=\frac{d \ln G(a)}{d \ln a}=-\frac{(1+z)}{G(z)} \frac{d G(z)}{d z} .
$$

As the growth factor $G(z)$ is degenerate with the HI bias factor, here we focus on the growth rate obtained from the redshift space distortion, and will discuss the measurement error on $f(z)$.

The redshift space power spectrum measured from the $21 \mathrm{~cm}$ intensity mapping could also be used as a test for gravity (Hall et al. 2013; Masui et al. 2010), or provide extra information on the dark energy if general relativity is assumed. For dark energy models, the growth rate can be parameterized as $f(z)=\Omega_{\mathrm{m}}^{\gamma}(z)$, with $\gamma_{\Lambda \mathrm{CDM}} \approx 0.55$ for the $\Lambda \mathrm{CDM}+\mathrm{GR}$ model. The value of $\gamma$ in other dark energy models with $w$ different from -1 does not deviate from $\gamma_{\Lambda \mathrm{CDM}}$ significantly.

The intensity mapping observation directly measures the $21 \mathrm{~cm}$ brightness temperature, and the measured $21 \mathrm{~cm}$ power spectrum, $P_{\Delta T}(\vec{k})=\bar{T}_{\mathrm{sig}}^{2} P_{\text {obs }}(\vec{k})$, is the power spectrum of brightness temperature $\delta T_{b}$ due to the $21 \mathrm{~cm}$ emission, in which the average signal temperature $\bar{T}_{\text {sig }}$ has been estimated (Barkana \& Loeb 2007; Chang et al. 2008; Seo et al. 2010) to be

$$
\bar{T}_{\mathrm{sig}}=190 \frac{x_{\mathrm{HI}}(z) \Omega_{\mathrm{H}, 0} h(1+z)^{2}}{H(z) / H_{0}} \mathrm{mK}
$$

where $x_{\mathrm{HI}}(z)$ is the neutral fraction of hydrogen at redshift $z$, and $\Omega_{\mathrm{H}, 0}$ is the ratio of the hydrogen mass density to the critical density at $z=0$. 
After the completion of cosmic reionization, the HI gas in the Universe is mostly distributed in galaxies hosted by halos. Therefore, we model the HI bias factors as halo bias factors weighted by the neutral hydrogen mass hosted by these halos (Gong et al. 2011):

$$
b_{i}^{\mathrm{HI}}(z)=\frac{\int_{M_{\min }}^{M_{\max }} d M n(M, z) M_{\mathrm{HI}}(M) b_{i}(M, z)}{\rho_{\mathrm{HI}}},
$$

for $i=1$ and 2, where $\rho_{\mathrm{HI}}$ is the mass density of HI gas, $n(M, z)$ is the halo mass function for which we use Sheth \& Tormen's formalism (Sheth \& Tormen 1999), $M_{\mathrm{HI}}(M)$ is the HI mass in a halo of mass $M$, and $b_{1}(M, z)$ and $b_{2}(M, z)$ are halo bias parameters. The mass density of HI clouds is given by

$$
\rho_{\mathrm{HI}}=\int_{M_{\min }}^{M_{\max }} d M n(M, z) M_{\mathrm{HI}}(M) .
$$

Following Gong et al. (2011), we take $M_{\text {min }}=10^{8} \mathrm{~h}^{-1} M_{\odot}$ for halos to retain their neutral gas (Loeb \& Barkana 2001), and take $M_{\max }=10^{13} \mathrm{~h}^{-1} M_{\odot}$ for the gas to have sufficient time to cool and form galaxies.

As for the relation between the $\mathrm{HI}$ gas mass $M_{\mathrm{HI}}$ and the host halo mass $M$, we use the fitting result by Gong et al. (2011), which is based on numerical simulation and consistent with observations:

$$
M_{\mathrm{HI}}(M)=A \times\left(1+\frac{M}{c_{1}}\right)^{b}\left(1+\frac{M}{c_{2}}\right)^{d}
$$

for $M>10^{10} M_{\odot}$, and $M_{\mathrm{HI}}(M)=X_{\mathrm{HI}}^{\text {gal }}\left(\Omega_{\mathrm{b}} / \Omega_{\mathrm{m}}\right) M$ with $X_{\mathrm{HI}}^{\text {gal }}=0.15$ for $M \leq 10^{10} M_{\odot}$. The best-fit parameters are $A=2.1 \times 10^{8}, c_{1}=1.0 \times 10^{11}, c_{2}=4.55 \times 10^{11}, b=2.65$, and $d=-2.64$ for redshift $z=1$. As the $M_{\mathrm{HI}}-M$ relation does not change much from $z=1$ to $z=3$ (Gong et al. 2011), we use the fixed values of these parameters throughout our calculation.

The halo bias factors can be obtained from the halo model (see Coorav \& Sheth 2002 for a review). The linear and the first non-linear bias factors of halos are (Scoccimarro et al. 2001; Mo et al. 1997)

$$
\begin{gathered}
b_{1}(M, z)=1+\epsilon_{1}+E_{1}, \\
b_{2}(M, z)=2\left(1+a_{2}\right)\left(\epsilon_{1}+E_{1}\right)+\epsilon_{2}+E_{2},
\end{gathered}
$$

where

$$
\epsilon_{1}=\frac{q \nu-1}{\delta_{\mathrm{sc}}(z)}, \epsilon_{2}=\frac{q \nu}{\delta_{\mathrm{sc}}(z)}\left(\frac{q \nu-3}{\delta_{\mathrm{sc}}(z)}\right)
$$

and

$$
E_{1}=\frac{2 p / \delta_{\mathrm{sc}}(z)}{1+(q \nu)^{p}}, \frac{E_{2}}{E_{1}}=\frac{1+2 p}{\delta_{\mathrm{sc}}(z)}+2 \epsilon_{1} .
$$

Here $a_{2}=-17 / 21, \nu \equiv \delta_{\mathrm{sc}}^{2}(z) / \sigma^{2}(M)$, and $\delta_{\mathrm{sc}}(z)=1.686 / G(z)$ is the critical overdensity required for spherical collapse at $z$, extrapolated to the present time using linear theory. For Sheth \& Tormen's halo mass function (Sheth \& Tormen 1999), $p \approx 0.3$, and $q=0.707$. 


\subsection{Generalized Noise Power Spectrum}

The fundamental observable of a radio interferometer is the visibility, which is the correlation between outputs of two receivers for a given baseline. For a given sky brightness distribution $I(\hat{n}, \nu)$, where $\hat{n}$ and $\nu$ are the sky position and the observing frequency respectively, the corresponding visilibity, in units of flux density, can be written as the Fourier transform of the sky brightness weighted by the beam pattern $A(\hat{n})$ of the two receivers:

$$
\begin{aligned}
V_{\alpha \beta,[\mathrm{Jy}]}\left(\vec{u}_{\alpha \beta}, \nu\right) & =\int d^{2} \hat{n} e^{-i 2 \pi \hat{n} \cdot \vec{u}_{\alpha \beta}} A_{\alpha}(\hat{n}, \nu) A_{\beta}^{*}(\hat{n}, \nu) I(\hat{n}, \nu) \\
& \approx \int d^{2} \hat{n} e^{-i 2 \pi \hat{n} \cdot \vec{u}_{\perp}} A_{\alpha}(\hat{n}, \nu) A_{\beta}^{*}(\hat{n}, \nu) I(\hat{n}, \nu),
\end{aligned}
$$

where $\vec{u}_{\alpha \beta}$ denotes the baseline vector in units of wavelength. Here in the second equality, we have used the flat-sky approximation, and $\vec{u}_{\perp}$ is the component of $\vec{u}_{\alpha \beta}$ perpendicular to the line of sight. In a large-scale survey like Tianlai, the flat-sky assumption will certainly break down, a full-sky representation based on the spherical harmonic expansion has been developed (Shaw et al. 2014). Here we use the flat-sky approximation and Fourier expansion, as for forecasting it is still sufficient.

For radio interferometers, it is convenient to define the equivalent visibility in units of brightness temperature, using the Rayleigh-Jeans approximation, so that

$$
V_{\alpha \beta,[\mathrm{K}]}\left(\vec{u}_{\perp}, \nu\right)=\int d^{2} \hat{n} e^{-i 2 \pi \hat{n} \cdot \vec{u}_{\perp}} A_{\alpha}(\hat{n}, \nu) A_{\beta}^{*}(\hat{n}, \nu) \delta T_{b}(\hat{n}, \nu) .
$$

The thermal noise of the measurement can be written as

$$
\delta V_{\alpha \beta,[\mathrm{K}]}\left(\vec{u}_{\perp}, \nu\right)=\frac{\lambda^{2} T_{\mathrm{sys}}}{A_{e} \sqrt{\Delta \nu t_{\vec{u}}}},
$$

where $\Delta \nu$ is the observed full bandwidth, $t_{\vec{u}}$ is the integration time of this baseline, $T_{\text {sys }}$ is the system temperature per polarization (we assume $T_{\mathrm{sys}}=50 \mathrm{~K}$ in this paper), and $A_{e}$ is the effective collecting area of each element. We can make a further Fourier transform of the visibility with respect to $\nu$, to obtain the so called visibility delay-spectrum(Parsons et al. 2012),

$$
V_{\alpha \beta,[\mathrm{K} \cdot \mathrm{MHz}]}\left(\vec{u}_{\perp}, u_{\|}\right)=\int d \nu e^{-i 2 \pi \nu u_{\|}} V_{\alpha \beta,[\mathrm{K}]}\left(\vec{u}_{\perp}, \nu\right)
$$

Now the three-dimensional vector $\vec{u} \equiv\left\{\vec{u}_{\perp}, u_{\|}\right\}$is the Fourier conjugate of the sky position vector $\vec{\theta}=\{\hat{n}, \nu\}$. The thermal noise in this representation is then (Morales 2005)

$$
\Delta T_{N}(\vec{u})=\frac{T_{\text {sys }}}{\sqrt{\Delta \nu t_{\vec{u}}}}\left(\frac{\lambda^{2} \Delta \nu}{A_{e}}\right)
$$

Here the factor $\lambda^{2} \Delta \nu / A_{e}$ represents the Fourier space resolution of the observation in the sense that any two vectors within it will be highly correlated. 
To extract cosmological information, one is interested in the correlation function of the visibilities measured at discrete baselines $\vec{u}_{i}$ and $\vec{u}_{j}$. If we neglect the correlation of thermal noise errors between measurements, the noise covariance matrix for visibilities is approximately diagonal, and can be written as (McQuinn et al. 2006; Bharadwaj \& Pandey 2003)

$$
\mathbf{C}^{N}\left(\vec{u}_{i}, \vec{u}_{j}\right)=\left\langle\Delta T_{N}\left(\vec{u}_{i}\right) \Delta T_{N}^{*}\left(\vec{u}_{j}\right)\right\rangle=\left(\frac{\lambda^{2} T_{\mathrm{sys}} \Delta \nu}{A_{e}}\right)^{2} \frac{\delta_{i j}}{\Delta \nu t_{\vec{u}}} .
$$

The integration time for baseline $\vec{u}$ can be written as

$$
t_{\vec{u}}=\frac{A_{e}}{\lambda^{2}} n\left(\vec{u}_{\perp}\right) t_{\mathrm{int}},
$$

where $n\left(\vec{u}_{\perp}\right)$ is the baseline number density of the interferometer in $u-v$ plane, and $A_{e} / \lambda^{2} \approx \delta u \delta v$ is the $\vec{u}$-space resolution. For an observation with survey area $\Omega_{\text {map }}$ larger than the field of view $\Omega_{\mathrm{FOV}}$ and uniform survey coverage, the integration time of each pointing $t_{\text {int }}=t_{\text {tot }}\left(\Omega_{\mathrm{FOV}} / \Omega_{\mathrm{map}}\right)$.

The the sample variance contribution to the covariance matrix is (McQuinn et al. 2006)

$$
\begin{aligned}
\mathbf{C}^{S V}\left(\vec{u}_{i}, \vec{u}_{j}\right)=\left\langle\delta T_{b}\left(\vec{u}_{i}\right) \delta T_{b}^{*}\left(\vec{u}_{j}\right)\right\rangle & \approx \delta_{i j} \int d^{3} \vec{u}\left|R\left(\vec{u}_{i}-\vec{u}\right)\right|^{2} P_{\Delta T}(\vec{u}) \\
& \approx \delta_{i j} \frac{\lambda^{2} \Delta \nu^{2}}{r_{a}^{2}(z) \Delta r(z) A_{e}} P_{\Delta T}\left(\vec{k}_{i \perp}, k_{i \|}\right),
\end{aligned}
$$

where $P_{\Delta T}$ is the $21 \mathrm{~cm}$ signal power spectrum. Here $R\left(\vec{u}_{i}-\vec{u}\right)$ is the response function for a given baseline $\vec{u}_{i}$, which is defined as the Fourier transform of the primary beam $A_{\alpha}(\hat{n}, \nu) A_{\beta}^{*}(\hat{n}, \nu)$ in Eq. (15). The Kronecker $\delta_{i j}$ arises due to the choice of the pixel size that is approximately the same as the support of function $R(\vec{u})$. The integration of $|R|^{2}$ then introduces a factor approximately equals the inverse of the Fourier space resolution, $\lambda^{2} \Delta \nu / A_{e}$, due to the normalization of $R(\vec{u})$. Here $\Delta r=y(z) \Delta \nu$ is the spatial resolution corresponding to bandwidth $\Delta \nu$. The comoving angular diameter distance $r_{a}(z)$ and the factor $y(z)=\lambda_{21}(1+z)^{2} / H(z)$ are used to convert the power spectrum from $\vec{u}$-space to the comoving $\vec{k}$-space:

$$
\vec{u}_{\perp}=\frac{r_{a}(z) k_{\perp}}{2 \pi}, \quad u_{\|}=\frac{y(z) k_{\|}}{2 \pi} .
$$

Given the total covariance matrix $\mathbf{C}=\mathbf{C}^{N}+\mathbf{C}^{S V}$, one could then estimate the measurement uncertainty of the bandpower from the Fisher matrix

$$
F_{a b}=\operatorname{Tr}\left[\mathbf{C}^{-1} \frac{\partial \mathbf{C}}{\partial p_{a}} \mathbf{C}^{-1} \frac{\partial \mathbf{C}}{\partial p_{b}}\right]
$$

where parameter $p_{a}$ is the bandpower $p_{a}=P_{\Delta T}\left(\vec{k}_{a}\right)$. For diagonal $\mathbf{C}$, the measurement error $\delta P_{\Delta T}$ is

$$
\delta P_{\Delta T}\left(\vec{k}_{i}\right)=\frac{1}{\sqrt{N_{c}}\left(\vec{k}_{i}\right)} \frac{A_{e} r_{a}^{2} \Delta r}{\lambda^{2} \Delta \nu^{2}}\left[C^{N}\left(\vec{k}_{i}, \vec{k}_{i}\right)+C^{S V}\left(\vec{k}_{i}, \vec{k}_{i}\right)\right]=\frac{1}{\sqrt{N_{c}}\left(\vec{k}_{i}\right)}\left[P^{N}\left(\vec{k}_{i}\right)+P^{S V}\left(\vec{k}_{i}\right)\right] .
$$


where the number of modes $N_{c}(k)=k_{\perp} d k_{\perp} d k_{\|} V /(2 \pi)^{2}$, with $V$ being the survey volume. Here we have denoted the signal power spectrum in the sample variance term as the sample variance power spectrum, i.e. $P^{S V}\left(\vec{k}_{i}\right)=P_{\Delta T}\left(\vec{k}_{i}\right)$, and the noise power spectrum $P^{N}(\vec{k})$ is

$$
P^{N}(k, z)=\frac{4 \pi f_{\mathrm{sky}} \lambda^{2} T_{\mathrm{sys}}^{2} y(z) r_{a}(z)^{2}}{A_{e} \Omega_{\mathrm{FOV}} t_{\mathrm{tot}}}\left(\frac{\lambda^{2}}{A_{e} n\left(\vec{k}_{\perp}\right)}\right),
$$

where $f_{\text {sky }}$ is the fraction of the sky coverage, i.e. $f_{\text {sky }}=\Omega_{\text {map }} / 4 \pi$, and $\Omega_{\mathrm{FOV}}$ is the field-of-view of a single pointing.

\subsection{Tianlai Noise Power Spectra}

We first calculate the baseline distribution function $n\left(\vec{u}_{\perp}\right)$ of the interferometer. In a real interferometer, for a pair of antennae with separation $\vec{u}$, the output is actually the average of the visibility on a region of the $\mathrm{u}-\mathrm{v}$ plane centered at $\vec{u}$. Instead of the discrete histogram, therefore, we incorporate the response function of an antenna pair $R(\vec{u})$ (Ansari et al. 2012) and derive a continuous function $n\left(\vec{u}_{\perp}\right)$, with the caveat that only $n\left(\vec{u}_{\perp}\right)\left(A_{e} / \lambda^{2}\right)$ is physically meaningful in this formalism. For the Tianlai cylinder array with receivers fixed along the focal lines of cylinders, the pair response pattern of a cylinder can be approximated as a two-dimensional triangular function with rectangular support (Thompson et al. 2001; Ansari et al. 2008, 2012), which is set by the cylinder width $W$ in east-west direction, $\Delta u_{W}=W / \lambda$, and the feed length $L$ in north-south direction, $\Delta u_{L}=L / \lambda$ :

$$
R\left(\vec{u}_{\perp}\right)=\left(\frac{\lambda^{2}}{A_{e}}\right) \Lambda\left(\frac{u_{L}}{\Delta u_{L}}\right) \Lambda\left(\frac{u_{W}}{\Delta u_{W}}\right)
$$

Here the triangular function $\Lambda(x)$ is defined as $1-|x|$ for $|x|<1$ and 0 otherwise. The baseline number density $n\left(\vec{u}_{\perp}\right)$ could be obtained simply by summing up $R(\vec{u})$ for all baselines, i.e.

$$
n\left(\vec{u}_{\perp}\right)=\sum_{i}^{n_{b}} R\left(\vec{u}_{\perp}-\vec{u}_{\perp}^{i}\right) .
$$

The baseline number density $n\left(\vec{u}_{\perp}\right)$ is normalized that the half-plane integral would give the total baseline number of $n_{b}=n_{r}\left(n_{r}-1\right) / 2$, where $n_{r}$ is the total number of receivers.

In the left panel of Fig. 1, we plot the baseline distribution $n(r)$ of different configurations of Tianlai. Besides the baseline distance $r$ in the interferometer frame, we also show on the upper abscissa the cosmological scales that the array could probe at frequency $\nu=750 \mathrm{MHz}$. Given the same cylinder dimension of pathfinder and its upgrade, the baseline densities of these two configurations drop at similar scales, around $20 \mathrm{~m}$. However, the larger feeds number of the pathfinder+ would increase the number of baselines available at a given scale. For the full-scale Tianlai, both the numbers of shorter and longer baselines are increased, therefore, the sensitivity will be improved significantly. 

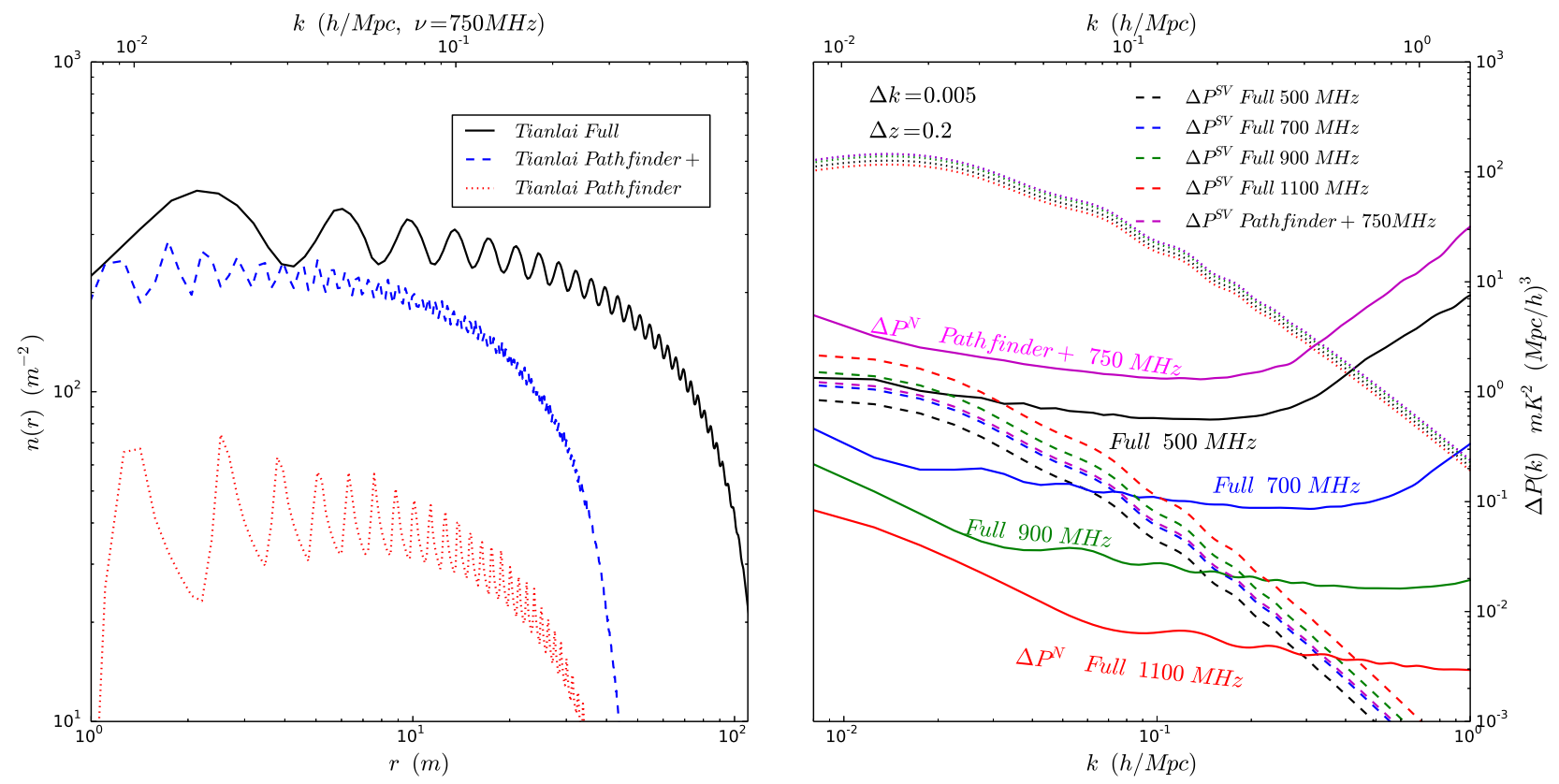

Fig. 1.- (Left): one-dimensional baseline distribution $n(r)$ of various Tianlai configurations, calculated from Eq. (28). Here x-axis $r$ is the physical distance in the interferometer frame, we also display the corresponding cosmological scale it probes at frequency $\nu=750 \mathrm{MHz}$ on the upper abscissa. (Right): The measurement error contributed from thermal noise, $\Delta P^{N}(k)=$ $P^{N}(k) / \sqrt{N_{c}(k)}$ (solid lines), and that from sample variance, $\Delta P^{S V}(k)=P^{S V}(k) / \sqrt{N_{c}(k)}$ (dashed lines). The case for Tianlai pathfinder+ is shown as the magenta lines, while the cases for fullscale Tianlai at various frequencies are shown as other colors. The $21 \mathrm{~cm}$ signal power spectra, $P_{\Delta T}(k)$, at corresponding redshifts are shown by dotted lines with corresponding colors, assuming a constant HI fraction $x_{\mathrm{HI}}=0.008$ at all redshifts. Here we adopt a wavenumber bin width of $\Delta k=0.005 \mathrm{Mpc} / \mathrm{h}$, and $\Delta z=0.2$.

In the right panel of Fig. 1, we plot the measurement error on the power spectrum due to thermal noise $\Delta P^{N}(k)=P^{N}(k) / \sqrt{N_{c}(k)}$ (solid lines) and sample variance $\Delta P^{S V}(k)=P^{S V}(k) / \sqrt{N_{c}(k)}$ (dashed lines). While only the errors at the medium frequency $f=750 \mathrm{MHz}$ are shown (the magenta set of lines) for the pathfinder+, we display four different frequencies from $500 \mathrm{MHz}$ to $1100 \mathrm{MHz}$ (from top to bottom for the thermal noise, and from bottom to top for the sample variance) for the full-scale survey. For the sample variance power spectra, we adopt a conservative assumption that $x_{\mathrm{HI}}=0.008$ at all redshifts. For comparison, we also plot the $21 \mathrm{~cm}$ signal power spectra, $P_{\Delta T}(k)$, at the corresponding redshifts with the dotted lines.

From the figure, we can see that for Tianlai pathfinder+, the thermal noise will dominate over the sample variance at all scales. This is also true for the high-redshift observation of the full-scale Tianlai, but the thermal noise gradually decreases towards lower redshift. At $z \sim 1$ (Full 700MHz), 
the two contributions are comparable at the BAO scale.

\subsection{The Power Spectrum with Expected Tianlai Errors}

As discussed in Sec2.2, the measurement error of the power spectrum is a sum of the sampling error and thermal noise. Since the measured $21 \mathrm{~cm}$ power spectrum is proportional to the HI power spectrum by a factor of $\bar{T}_{\text {sig }}^{2}$, we use the measurement error on the HI power spectrum for the Fisher forecasts in the following sections, and it is

$$
\Delta P_{\mathrm{obs}}(\vec{k})=\frac{1}{\sqrt{N_{c}}}\left[P_{\mathrm{obs}}(\vec{k})+N(k)\right]
$$

where $N(k)$ is related to the thermal noise power by $P^{N}=\bar{T}_{\text {sig }}^{2} N(k)$, and $N_{c}$ is the number of independent modes in that pixel in Fourier space as discussed in section 2.2.
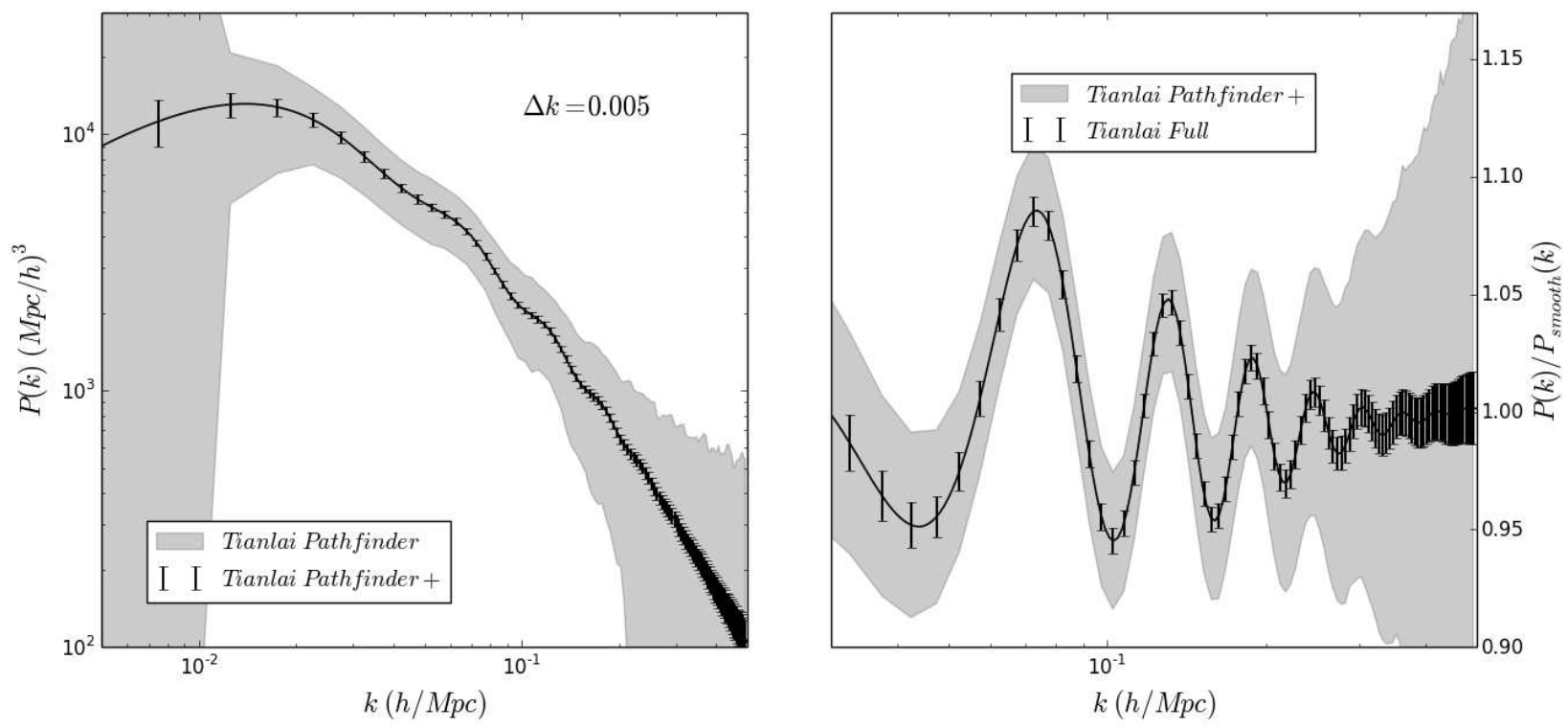

Fig. 2.- Left panel: the measurement errors on the power spectrum at $z=1$ for the Tianlai pathfinder (shaded area) and the pathfinder+ (error bars). Right panel: the relative one with respect to the smooth power spectrum with errors expected from the Tianlai pathfinder+ (shaded area) and the full-scale Tianlai (error bars). The assumed survey area is $10000 \mathrm{deg}^{2}$, and the integration time is 1 year. The wavenumber bin width for this plot is $\Delta k=0.005 \mathrm{Mpc} / \mathrm{h}$.

The instantaneous field of view of a cylindrical radio telescope is narrow in right ascension but very broad in declination, limitted primarily by the illumination angle of the feeds. The rotation of the Earth results in a broad coverage in right ascension. The illumination angle of the feeds of Tianlai is about $120^{\circ}$. Assuming a latitude of $\theta_{\text {lat }}=44^{\circ}$, the Tianlai array covers the declination 
angle from $-16^{\circ}$ to $90^{\circ}$. Considering the masking effects in order to avoid the disk area of the Milky Way and bright radio sources, we conservatively assume a survey area of $10000 \mathrm{deg}^{2}$ throughout.

The left panel of Fig. 2 shows the power spectrum at $z=1$ with measurement errors expected from the Tianlai pathfinder (shaded area) and the pathfinder+ (error bars), while the right panel shows the relative power spectrum with respect to the smooth power spectrum with errors expected from the Tianlai pathfinder+ (shaded area) and the full-scale Tianlai (error bars) respectively. The integration time is assumed to be 1 year. Note that the error bar for $P(k)$ depends on the binning of $k$. Here we have chosen a bin width of $\Delta k=0.005 \mathrm{Mpc} / \mathrm{h}$. If a different bin width is chosen, the error bar on power spectrum can be obtained by scaling, but the constraints on our interested parameters are insensitive to the choice of binning.

\section{Fisher forecast on the constraint on dark energy}

From the power spectrum measurement at a given redshift, the Fisher information matrix can be written as (Tegmark 1997; Seo \& Eisenstein 2003; Mao et al. 2008)

$$
F_{\alpha \beta}=\sum_{k}\left[\frac{\partial P_{\mathrm{obs}}(\vec{k})}{\partial \alpha} \frac{\partial P_{\mathrm{obs}}(\vec{k})}{\partial \beta}\right] /\left[\Delta P_{\mathrm{obs}}(\vec{k})\right]^{2} .
$$

Here the free parameters $\alpha$ and $\beta$ are taken from $\left\{D_{\mathrm{A}}\left(z_{i}\right), H\left(z_{i}\right), b_{1, i}^{\mathrm{HI}}, f\left(z_{i}\right), P_{\text {shot,i }}\right\}$ for each redshift bin $z_{i}$. The nuisance parameters in the model $\left\{b_{1, i}^{\mathrm{HI}}, P_{\text {shot,i }}\right\}$ can be marginalized by selecting the submatrix of $F_{\alpha \beta}^{-1}$ with only the appropriate columns and rows. We can then derive the measurement errors on the expansion and structure growth history parameters.

For the Tianlai pathfinder and pathfinder+ experiment, the observation frequency range is $700-800 \mathrm{MHz}$, and we divide this frequency band into 3 bins with equal bandwidth, and obtain estimates of measurement error for the corresponding redshift bins. For the planned full-scale Tianlai experiment, the frequency range of $400-1420 \mathrm{MHz}$ is equally divided into 8 bins, the bin size in this latter case is larger than in the pathfinder case, but the bin size $\Delta z$ does not really matter in the end.

We plot the measurement errors on the angular diameter distance $D_{\mathrm{A}}\left(z_{i}\right)$, the Hubble expansion rate $H\left(z_{i}\right)$, and the growth rate $f\left(z_{i}\right)$ in the left, central, and right panels of Fig. 3, respectively, for the pathfinder+ (blue error bars) and the full-scale Tianlai experiments (red error bars). The integration time is assumed to be 1 year in all cases. We see that the pathfinder + experiment can obtain a useful measurement on $D_{A}(z)$ and $H(z)$ at $z=1$. For the growth rate $f$, the errors

are larger, but still a useful check against certain modified gravity models could be obtained. The full-scale experiment can offer good precision throughout the interested parameter ranges.

From the cosmographic measurments $D_{A}(z), H(z), f(z)$, one can constrain the cosmological model parameters. In this paper, we consider a redshift-dependent equation of state for the dark 

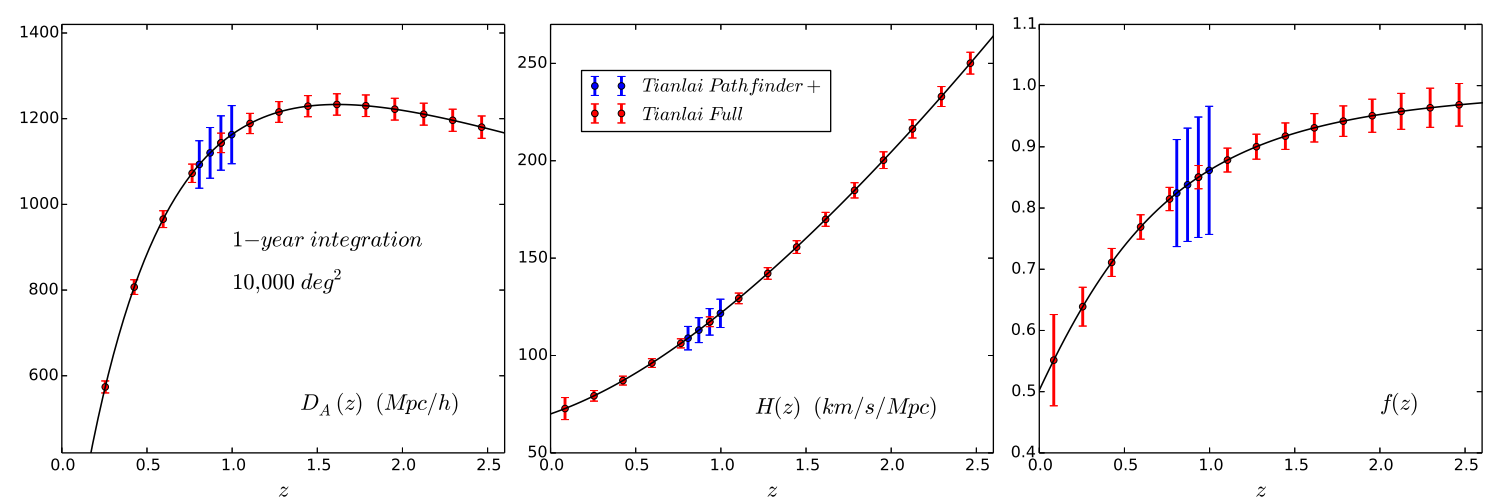

Fig. 3.- Measurement errors on the angular diameter distance $D_{\mathrm{A}}$ (left panel), the Hubble expansion rate $H$ (central panel), and the growth rate $f(z)=d \ln G / d \ln a$ (right panel). The integration time is assumed to be 1 year. Note that here the number of redshift bins is larger than the one we used for forecasting the constraint on dark energy equation of state, just to make the error bars visible.

energy parameterized in the form of

$$
w(z)=w_{0}+w_{a}[1-a(z)]=w_{0}+w_{a} \frac{z}{1+z} .
$$

The Fisher matrix of dark energy parameters $w_{0}$ and $w_{a}$ is obtained by converting from the parameter space $\left\{p_{\alpha}\right\}=\left\{D_{\mathrm{A}, \mathrm{i}}, H_{i}, f_{i}\right\}$ to the dark energy parameter space $\left\{q_{m}\right\}=\left\{w_{0}, w_{a}, \Omega_{X}\right\}$, using

$$
F_{m n}^{D E}=\sum_{\alpha, \beta} \frac{\partial p_{\alpha}}{\partial q_{m}} F_{\alpha \beta}^{\text {dis }} \frac{\partial p_{\beta}}{\partial q_{n}} .
$$

To help break the parameter degeneracy between parameters, we combine the BAO data from Tianlai intensity mapping observation with the data obtained from CMB observations. The total Fisher matrix is given by (Wang et al. 2009)

$$
F_{\alpha \beta}^{t o t}=F_{\alpha \beta}^{C M B}+\sum_{i} F_{\alpha \beta}^{I M}\left(z_{i}\right)
$$

where $F_{\alpha \beta}^{I M}\left(z_{i}\right)$ is the Fisher matrix derived from the $i$-th redshift bin of the large scale structure imtensity mapping, and $F_{\alpha \beta}^{C M B}$ is the CMB Fisher matrix.

The 1- $\sigma$ and 2- $\sigma$ measurement error contours for the variable dark energy equation of state parameters $w_{0}$ and $w_{a}$ are shown in Fig. 4 for the full-scale Tianlai experiment. Here we have assumed that the frequency range probed is $400-1420 \mathrm{MHz}$, the usable survey area is $10000 \mathrm{deg}^{2}$, and the integration time is 1 year. The measurement error is obtained with a joint constraint with the CMB data, but no other observational data. We expect $\sigma_{w_{0}} \approx 0.0815$ and $\sigma_{w_{a}} \approx 0.210$. This is comparable with the precision of stage IV dark energy experiments as defined in the Dark Energy Task Force (DETF) report (Albrecht et al. 2006). 


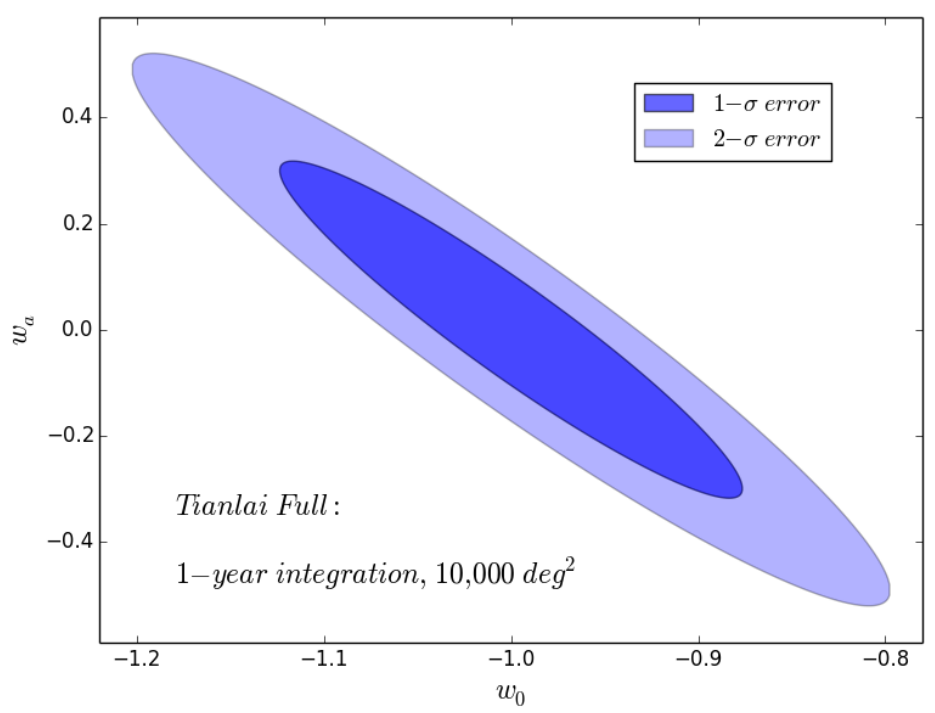

Fig. 4.- Constraints on dark energy equation of state parameters $w_{0}$ and $w_{a}$ from full-scale Tianlai experiments. The two contours are for $1-\sigma$ and $2-\sigma$ constraints respectively. The integration time is 1 year, and the survey area is assumed to be $10000 \mathrm{deg}^{2}$.

\section{Fisher Forecasts for the Primordial Non-Gaussianity}

Typical single field slow roll inflation models predict that the primordial density fluctuations follow the Gaussian distribution, though the density distribution deviates from Gaussianity as the structures grow and non-linearities appear. Detection or constraint on the primordial nonGaussianity will provide invaluable information on the origin of the Universe.

Compared with the observable galaxies which correspond to high density peaks of the matter density distribution, the neutral hydrogen gas that exists in galaxies of almost all mass scales is a less biased tracer of the underlying matter density, allowing the primordial non-Gaussianity to be investigated from a different perspective.

The non-Gaussianity of the primordial density fluctuations can induce a scale-dependent and redshift-dependent HI bias, similar to other biased tracers (Dalal et al. 2008; Matarrese \& Verde 2008). This effect can be used to constrain the primordial non-Gaussianity. Camera et al. (2013) has demonstrated that a small but compact array working at $\sim 400 \mathrm{MHz}$ would be possible to place tight constraints on the $f_{\mathrm{NL}}$, with an error close to $\sigma_{f_{\mathrm{NL}}} \sim 1$. We shall make forecast for making such constraints with the Tianlai experiment.

Once the large scale structure of the $21 \mathrm{~cm}$ brightness temperature fluctuations are mapped out, this same set of data can also be used to measure the bispectrum of HI gas distribution. The HI bispectrum consists contributions from primordial non-Gaussianity, the non-linear gravity evolution, and the non-linear HI bias. The relative importance of primordial non-Gaussianity 
increases toward higher redshifts (Sefusatti \& Komatsu 2007; Jeong \& Komatsu 2009). The 21 $\mathrm{cm}$ experiment can in principle observe the large scale structure at relatively high redshifts from the ground without being affected significantly by the atomosphere, and this is an advantage of this method, though at present the $21 \mathrm{~cm}$ observation is still limited to lower redshifts than the optical observations. The $21 \mathrm{~cm}$ intensity mapping is much more efficient with large survey volume without resolving individual galaxies. Using the $21 \mathrm{~cm}$ bispectrum from the dark ages, Pillepich et al. (2007) found that very low frequency radio observations with high angular resolution could potentially detect primordial non-Gaussianity with $f_{\mathrm{NL}} \sim 1$. Here we focus on the HI bispectrum after reionization, and assess the constraining power of the $21 \mathrm{~cm}$ bispectrum measured by Tianlai experiment.

\subsection{Constraints on $f_{\mathrm{NL}}$ from the HI Power Spectrum}

The non-Gaussianity in the primordial density fluctuations can result in a scale-dependence in the halo bias, which originates from coupling between large and small scales modes (Dalal et al. 2008; Matarrese \& Verde 2008). For the standard local type primordial non-Gaussianity, the scale-dependent non-Gaussian correction to the linear halo bias, to the leading order, is (see e.g. Desjacques et al. 2011; Adshead et al. 2012; D'Aloisio et al. 2013)

$$
\Delta b^{d}(k, z)=\frac{2 f_{\mathrm{NL}}\left(b_{1}^{\mathrm{G}}-1\right) \delta_{c}}{\mathcal{M}(k, z)}
$$

where $b_{1}^{\mathrm{G}}$ is the linear halo bias for the Gaussian density field, $\delta_{c}=1.686$ is the critical overdensity for spherical collapse, and $\mathcal{M}(k, z)$ relates the density fluctuations in Fourier space, $\delta_{k}$, to the primordial curvature perturbation, $\Phi_{k}$, via the Poisson equation:

$$
\delta_{k}(z)=\mathcal{M}(k ; z) \Phi_{k},
$$

where

$$
\mathcal{M}(k ; z)=\frac{2}{3} \frac{k^{2} T(k) G^{+}(z) c^{2}}{\Omega_{\mathrm{m}} H_{0}^{2}} .
$$

Here $T(k)$ is the matter transfer function normalized to unity on large scales, $c$ is the speed of light, and $G^{+}(z)=g(0) G(z)$ is the growth factor of the growing mode of density perturbations, in which $g(0)=\left(1+z_{\mathrm{i}}\right)^{-1} G^{-1}\left(z_{\mathrm{i}}\right)$ with $z_{\mathrm{i}}$ being the initial redshift, and $G(z)$ is the linear growth factor normalized to unity at $z=0$. The corrected linear halo bias is $b_{1}(k, M, z)=b_{1}^{\mathrm{G}}(M, z)+\Delta b^{d}(k, M, z)$.

The power spectrum of the density fluctuations of $\mathrm{HI}$ gas is $P_{\mathrm{HI}}(k, z)=\left[b_{1}^{\mathrm{HI}}(k, z)\right]^{2} P_{\mathrm{L}}(k, z)$, in which $P_{\mathrm{L}}(k, z)$ is the linear matter power spectrum, and the scale-dependent HI bias $b_{1}^{\mathrm{HI}}(k, z)$ is related to the corrected linear halo bias via the model described in section 2.1. The observed power spectrum in redshift space after averaging over angles in $k$ space is (Peacock 1997)

$$
P_{\mathrm{S}}(k, z)=a_{0}^{\mathrm{P}}(\beta) P_{\mathrm{HI}}(k, z),
$$


where $a_{0}^{\mathrm{P}}(\beta)=1+\frac{2}{3} \beta+\frac{1}{5} \beta^{2}$, with $\beta=\Omega_{\mathrm{m}}^{0.55}(z) / b_{1}^{\mathrm{HI}}$. We apply the same Fisher matrix as Eq. (30), but here we take $f_{\mathrm{NL}}$ as the single parameter, and fix all the other cosmological parameters.

When adding information from all available wavenumbers in the Fisher matrix, $k_{\max }$ is limited by the Nyquist frequency, $k_{\mathrm{Nyq}}=\pi /$ resolution, which arises from the non-zero beam size of the cylinder array (Seo et al. 2010), as well as by the non-linear wavenumber cutoff, $k_{\text {nonl }}$, above which the linear power spectra are not accurate. Here we adopt conservative values for $k_{\text {nonl }}$ by requiring $\sigma\left(R=\pi / 2 k_{\text {nonl }} ; z\right)=0.5$ at each redshift bin (Seo \& Eisenstein 2003). Therefore, $k_{\text {max }}=\operatorname{MIN}\left\{k_{\mathrm{Nyq}}, k_{\text {nonl }}\right\}$. Effectivley, $k_{\max }$ is limited by Nyquist wavenumber for pathfinder and pathfinder + , while for the full-scale Tianlai, $k_{\max }$ is mostly set by the non-linear cutoff, except for the highest redshift bin. On the other hand, $k_{\min }$ is set by the scale defined by the size of each redshift bin.

With the same survey parameters and redshift bins as in section 3 , we find that the constraint on the non-linear parameter $f_{\mathrm{NL}}$ for the local model is quite weak for the pathfinder and pathfinder+ experiments. With the full-scale Tianlai experiment, we can achieve $\sigma_{\mathrm{f}_{\mathrm{NL}}}^{\text {local }} \sim 14$. The exact numbers of the predicted $1-\sigma$ errors for Tianlai pathfinders and for the full-scale Tianlai are listed in table 2 .

Table 2: The predicted $1-\sigma$ errors of $f_{\mathrm{NL}}$ using HI power spectrum measured by Tianlai

\begin{tabular}{cccc}
\hline \hline & & & \\
& pathfinder & pathfinder + & full scale \\
\hline$N_{\text {feed }}$ per cylinder & 32 & 72 & 256 \\
\hline$\sigma_{\mathrm{f}_{\mathrm{NL}}}^{\text {local }}$ & 1504 & 161 & 14.1 \\
\hline
\end{tabular}

\subsection{Constraints on $f_{\mathrm{NL}}$ from the HI Bispectrum}

On large scales, the matter bispectrum is well described by the tree-level expression, and the loop corrections remain very small (Tasinato et al. 2013; Gong \& Takahashi 2014). Higher order terms such as the trispectrum could contribute significantly to the bispectrum of high density peaks (Sefusatti 2009; Jeong \& Komatsu 2009), but as the HI gas is much less biased than observable galaxies - for the Tianlai experiment, the HI bias is not far from 1 - we expect such contribution to be less significant, though the exact amount can not be obtained without going through the lengthy calculations. Here we neglect the higher order terms and account only for the tree level matter bispectrum here, and reserve the investigation of the contribution from matter trispectrum to the HI bispectrum to future works. If such contribution is significant, it would increase the HI bispectrum, and we would obtain a stronger constraint on $f_{\mathrm{NL}}$, so our current estimate may be regarded as a relatively conservative one. 
As we are interested in forecasting the constraining power of HI bispectrum observations on the primordial non-Gaussianity, i.e. the parameter $f_{\mathrm{NL}}$, in the following, we shall focus on the reduced HI bispectrum, $Q_{\mathrm{HI}}$, which is much less sensitive to other cosmological parameters (Sefusatti \& Komatsu 2007). In the real experiments, we always measure the $21 \mathrm{~cm}$ brightness temperature in redshift space. Similar to the tree-level expresion for the observed galaxy bispectrum (Sefusatti \& Komatsu 2007), the reduced HI bispectrum in redshift space after averaging over angles in $k$ space is

$$
Q_{s}\left(k_{1}, k_{2}, k_{3}\right)=\frac{a_{0}^{\mathrm{B}}(\beta)}{\left[a_{0}^{\mathrm{P}}(\beta)\right]^{2}}\left[\frac{1}{b_{1}^{\mathrm{HI}}} Q^{\mathrm{tree}}\left(k_{1}, k_{2}, k_{3}\right)+\frac{b_{2}^{\mathrm{HI}}}{\left(b_{1}^{\mathrm{HI}}\right)^{2}}\right],
$$

where $a_{0}^{\mathrm{B}}(\beta)=1+\frac{2}{3} \beta+\frac{1}{9} \beta^{2}$ converts the bispectrum from real space to redshift space, and $Q^{\text {tree }}$ is the reduced tree-level bispectrum of underlying matter. The first term includes the contributions from primordial non-Gaussianity and non-linear gravitational evolution, and the second represents the contribution from non-linear bias of HI gas.

The reduced matter bispectrum can be written as the sum of two contibutions:

$$
\begin{aligned}
Q^{\text {tree }}\left(k_{1}, k_{2}, k_{3}\right) & =Q_{\mathrm{I}}\left(k_{1}, k_{2}, k_{3}\right)+Q_{\mathrm{G}}\left(k_{1}, k_{2}, k_{3}\right) \\
& =\frac{B_{\mathrm{I}}\left(k_{1}, k_{2}, k_{3}\right)}{P_{\mathrm{L}}\left(k_{1}\right) P_{\mathrm{L}}\left(k_{2}\right)+(2 \text { perm. })}+\frac{B_{\mathrm{G}}\left(k_{1}, k_{2}, k_{3}\right)}{P_{\mathrm{L}}\left(k_{1}\right) P_{\mathrm{L}}\left(k_{2}\right)+(2 \text { perm. })},
\end{aligned}
$$

where $+($ n perm. $)$ stands for the sum of $n$ additional terms permuting $k_{1}, k_{2}$, and $k_{3}$. The matter bispectrum due to gravity alone, $B_{\mathrm{G}}$, is given by the second order perturbation theory (Fry) 1984; Bernardeau et al. 2002), and the the matter bispectrum contributed from primordial nonGaussianity, $B_{\mathrm{I}}$, is related to the bispectrum of curvature perturbations, $B_{\Phi}$, by

$$
B_{\mathrm{I}}\left(k_{1}, k_{2}, k_{3}\right)=\mathcal{M}\left(k_{1} ; z\right) \mathcal{M}\left(k_{2} ; z\right) \mathcal{M}\left(k_{3} ; z\right) B_{\Phi}\left(k_{1}, k_{2}, k_{3}\right) .
$$

We consider two models of primordial non-Gaussianity here, i.e. the local model and the equilateral model, but the same forecast can also be applied to other models of interest. The local model is physically motivated, in this case the contributions from the squeezed triangular configurations dominate. The leading contribution to the $f_{\mathrm{NL}}^{\text {local }}$ expansion of the bispectrum of curvature perturbation is

$$
\begin{aligned}
B_{\Phi}^{\text {local }}\left(k_{1}, k_{2}, k_{3}\right) & \simeq 2 f_{\mathrm{NL}}^{\text {local }}\left[P_{\Phi}\left(k_{1}\right) P_{\Phi}\left(k_{2}\right)+P_{\Phi}\left(k_{2}\right) P_{\Phi}\left(k_{3}\right)+P_{\Phi}\left(k_{3}\right) P_{\Phi}\left(k_{1}\right)\right] \\
& =2 f_{\mathrm{NL}}^{\text {local }} \Delta_{\Phi}^{2}\left[\frac{1}{k_{1}^{4-n_{s}} k_{2}^{4-n_{s}}}+(2 \text { perm. })\right]
\end{aligned}
$$

where $\Delta_{\Phi} \equiv P_{\Phi} / k^{n_{s}-4}$, and $P_{\Phi}(k)$ is the curvature power spectrum. The equilateral model is a good approximation to the higher derivative models (Creminelli 2003) and the DBI inflationary model(Alishahiha et al. 2004). The bispectrum of curvature perturbation for the equilateral model 
is

$$
\begin{aligned}
B_{\Phi}^{\text {equil. }}=6 f_{\mathrm{NL}}^{\text {equil. }} \Delta_{\Phi}^{2}[ & -\frac{1}{k_{1}^{4-n} k_{2}^{4-n} n_{s}}-\frac{1}{k_{2}^{4-n_{s}} k_{3}^{4-n_{s}}}-\frac{1}{k_{3}^{4-n_{s}} k_{1}^{4-n_{s}}}-\frac{2}{\left(k_{1} k_{2} k_{3}\right)^{2\left(4-n_{s}\right) / 3}} \\
& \left.+\left(\frac{1}{k_{1}^{\left(4-n_{s}\right) / 3} k_{2}^{2\left(4-n_{s}\right) / 3} k_{3}^{4-n_{s}}}+(5 \text { perm. })\right)\right] .
\end{aligned}
$$

Following Scoccimarro et al. (1998), a bispectrum estimator for a cubic survey volume of $V$ can be defined as

$$
\hat{B}\left(k_{1}, k_{2}, k_{3}\right) \equiv \frac{V_{\mathrm{f}}}{V_{\mathrm{B}}\left(k_{1}, k_{2}, k_{3}\right)} \int_{k_{1}} d^{3} \vec{q}_{1} \int_{k_{2}} d^{3} \vec{q}_{2} \int_{k_{3}} d^{3} \vec{q}_{3} \delta_{\mathrm{D}}\left(\vec{q}_{1}+\vec{q}_{2}+\vec{q}_{3}\right) \delta\left(\vec{q}_{1}\right) \delta\left(\vec{q}_{2}\right) \delta\left(\vec{q}_{3}\right),
$$

where $V_{\mathrm{f}} \equiv k_{\mathrm{f}}^{3}=(2 \pi)^{3} / V$ is the elemental volume in $k$ space of observation cells, and each integration is over the range $\left[k_{i}-\Delta k / 2, k_{i}+\Delta k / 2\right]$ centered on $k_{i}$, with $\Delta k$ equal to a multiple of $k_{\mathrm{f}}$. Here $\delta_{\mathrm{D}}\left(\vec{q}_{1}+\vec{q}_{2}+\vec{q}_{3}\right)$ is the Dirac delta function which ensures that the vectors $\vec{q}_{1}, \overrightarrow{q_{2}}$, and $\vec{q}_{3}$ form a triangle, while $V_{\mathrm{B}}\left(k_{1}, k_{2}, k_{3}\right)$ is the normalization factor given by

$$
V_{\mathrm{B}} \equiv \int_{k_{1}} d^{3} \vec{q}_{1} \int_{k_{2}} d^{3} \vec{q}_{2} \int_{k_{3}} d^{3} \vec{q}_{3} \delta_{\mathrm{D}}\left(\vec{q}_{1}+\vec{q}_{2}+\vec{q}_{3}\right) \simeq 8 \pi^{2} k_{1} k_{2} k_{3} \Delta k_{1} \Delta k_{2} \Delta k_{3}
$$

In the following, we assume $\Delta k_{i}=k_{\mathrm{f}}$ so as to take into account all "fundamental" triangular configurations.

To the leading order, the variance of this estimator is (Scoccimarro et al. 1998)

$$
\Delta B_{s}^{2}\left(k_{1}, k_{2}, k_{3}\right) \simeq(2 \pi)^{3} V_{\mathrm{f}} \frac{s_{123}}{V_{\mathrm{B}}} P_{\mathrm{tot}}\left(k_{1}\right) P_{\mathrm{tot}}\left(k_{2}\right) P_{\mathrm{tot}}\left(k_{3}\right),
$$

where $s_{123}=6,2,1$ respectively for equilateral, isosceles and general triangles, and $P_{\text {tot }}(k)$ is total measured power spectrum including the redshift space HI power spectrum, $P_{\mathrm{S}}(k)=a_{0}^{\mathrm{P}}(\beta) P_{\mathrm{HI}}(k)$, and the noise power spectrum $N(k)$.

The Fisher matrix for observations of reduced bispectrum at a given redshift bin can be written as

$$
F_{\alpha \beta} \equiv \sum_{k_{1}=k_{\min }}^{k_{\max }} \sum_{k_{2}=k_{\min }}^{k_{1}} \sum_{k_{3}=k_{\min }^{\star}}^{k_{2}} \frac{\partial Q_{s}}{\partial \alpha} \frac{\partial Q_{s}}{\partial \beta} \frac{1}{\Delta Q_{s}^{2}},
$$

where $\Delta Q_{s}^{2}$ is the variance of the reduced HI bispectrum measured in redshift space, and $\alpha$ and $\beta$ represent the parameters we are interested in, i.e. $f_{\mathrm{NL}}$, and $b_{1}^{\mathrm{HI}}\left(z_{i}\right)$ and $b_{2}^{\mathrm{HI}}\left(z_{i}\right)$ for each redshift bin $z_{i}$ of the survey. The three sums are over all combinations of $k_{1}, k_{2}$ and $k_{3}$ that form triangles, in steps of $\Delta k_{i}$, with $k_{\min }^{\star}=\max \left(k_{\min },\left|k_{1}-k_{2}\right|\right)$. In each redshift bin, we divide the survey volume into cubes, and $k_{\min }$ is still set by the scale spanning the redshift bin. $k_{\max }$ is set by the Nyquist frequency or the smallest scale at which we can trust our model for the HI bispectrum. Here we assume the tree-level bispectrum breaks down below the non-linear scale cutoff, so that $k_{\text {max }}=\operatorname{Min}\left\{k_{\mathrm{Nyq}}, k_{\text {nonl }}\right\}$. If we assume the variance of the HI bispectrum $\Delta B_{s}$ dominates over the 
variance of the HI power spectrum $\Delta P_{s}$, then the variance of the reduced HI bispectrum in redshift space can be written as (Sefusatti \& Komatsu 2007):

$$
\Delta Q_{s}^{2}\left(k_{1}, k_{2}, k_{3}\right) \simeq \frac{\Delta B_{s}^{2}\left(k_{1}, k_{2}, k_{3}\right)}{\left[P_{s}\left(k_{1}\right) P_{s}\left(k_{2}\right)+(2 \text { perm. })\right]^{2}},
$$

with the $\Delta B_{s}^{2}$ given by Eq. (45).

We assume the the fiducial values of HI bias parameters as given in section 2.1, and take the fiducial value of $f_{\mathrm{NL}}=0$ for both the local and equilateral models. Assuming 1 year's integration time and a total survey area of $10000 \mathrm{deg}^{2}$, the marginalized $1-\sigma$ errors on $f_{\mathrm{NL}}^{\text {local }}$ and $f_{\mathrm{NL}}^{\text {equil }}$ are listed in table 3. Again, we find that the pathfinder and pathfinder+ data are insufficient to provide much constraint on the bispectrum, due to the large error in its measurement. With the full-scale

Tianlai experiment, we could achieve $\sigma_{\mathrm{f}_{\mathrm{NL}}}^{\text {local }} \sim 22$ for the local model, and $\sigma_{\mathrm{f}_{\mathrm{NL}}}^{\text {equil }} \sim 157$ for the equilateral model.

\section{Conclusions}

In this work, we assess the capability of the Tianlai experiments in constraining various cosmological parameters, specifically the dark energy equation of state and the level of primordial non-Gaussianity. We use the Fisher information matrix method, which are widely used for making such forecasts. We have compared our results with other forecasts on $21 \mathrm{~cm}$ intensity mapping experiments (Chang et al. 2008; Ansari et al. 2008; Seo et al. 2010; Ansari et al. 2012; Alonso et al. 2014), and found they generally yield similar results when the same conditions are assumed.

Currently, our plan is to first test the principle and key technologies with a smaller scale pathfinder experiment, then upgrade to the pathfinder+ experiment, before eventually building the the full-scale Tianlai experiment. The goal of the pathfinders is to test the technologies and feasibility of HI intensity mapping observations with cylinder arrays, and as shown in this work, we expect to be able to measure the HI power spectrum with the pathfinders, but the constraints obtainable on cosmological parameters would be fairly weak.

The full-scale Tianlai experiment will significantly tighten the constraints by adding the number

Table 3. The marginalized $1-\sigma$ errors of $f_{\mathrm{NL}}$ using HI bispectrum measured by Tianlai

\begin{tabular}{|c|c|c|c|}
\hline$N_{\text {feed }}$ per cylinder & $\begin{array}{c}\text { Pathfinder } \\
32\end{array}$ & $\begin{array}{c}\text { Pathfinder }+ \\
72\end{array}$ & $\begin{array}{c}\text { Full scale } \\
256\end{array}$ \\
\hline$\sigma_{\mathrm{f}_{\mathrm{NL}}}^{\text {local }}$ & 70814 & 2272 & 21.7 \\
\hline$\sigma_{\mathrm{f}_{\mathrm{NL}}^{\text {equil }}}^{1 \mathrm{u}}$ & 79427 & 2754 & 157 \\
\hline
\end{tabular}


of receivers, hence increasing the effective collecting area of the cylinders, and by expanding the scale of the cylinders, hence increasing the spatial resolution. Assuming an integration time of 1 year, and a survey area of $10000 \mathrm{deg}^{2}$, we expect $\sigma_{w_{0}} \sim 0.082$ and $\sigma_{w_{a}} \sim 0.21$ from the BAO and RSD measurements. This is comparable to the expected precision from stage IV dark energy experiments as defined by the DETF report (Albrecht et al. 2006), while the cost would only be a small fraction of the such experiments.

The primordial non-Gaussianity can be constrained by looking for scale-dependent bias of the power spectrum, or by bispectrum measurement. We find $\sigma_{\mathrm{f}_{\mathrm{NL}}}^{\text {local }} \sim 14$ from the power spectrum measurements with scale-dependent bias, and $\sigma_{\mathrm{f}_{\mathrm{NL}}}^{\text {local }} \sim 22$ and $\sigma_{\mathrm{f}_{\mathrm{NL}}}^{\text {equil }} \sim 157$ from the bispectrum measurements. The constraints on the primordial non-Gaussianity from large scale structure observations including this one are generally weaker than from high precision CMB observations, but they probe different scales, so it is still very important to do such observations.

In making these forecasts, we have assumed that the foregrounds can be effectively removed, and their residuals only affect the overal system temperature. In fact, due to the complicated system responses and our imperfect knowledge of it from calibration, the foreground removal will not be so effective, and the foreground may also introduce non-Gaussian features which could potentially contaminate the measurement of the primordial non-Gaussianity. These problems may degrade the measurement precision (Ansari et al. 2012). Therefore, the results presented here should be regarded as an ideal case. We plan to make more detailed simulations to assese the effects of calibration and foreground subtraction on the measurement process for the Tianlai experiments in the near future.

We thank Fengquan Wu, Yi Mao, Hong Guo, Junqing Xia, and Kwan Chuen Chan for many helpful discussions. This work is supported by the MoST 863 program grant 2012AA121701, the NSFC grants 11373030 and 11303034, and the CAS Strategic Priority Research Program "The Emergence of Cosmological Structures" Grant No. XDB09000000.

\section{REFERENCES}

Abdalla, F. B., Blake, C., \& Rawlings, S. 2010, MNRAS, 401, 743

Adshead, P., Baxter, E. J., Dodelson, S., \& Lidz, A. 2012, Phys. Rev. D, 86, 063526

Albrecht, A., Bernstein, G., Cahn, R., et al. 2006, ArXiv Astrophysics e-prints, astro-ph/0609591

Alishahiha, M., Silverstein, E., \& Tong, D. 2004, Phys. Rev. D, 70, 123505

Alonso, D., Ferreira, P. G., \& Santos, M. G. 2014, MNRAS, 444, 3183

Anderson, L., Aubourg, É., Bailey, S., et al. 2014, MNRAS, 441, 24 
Ansari, R., Le Goff, J.-M., Magneville, C., et al. 2008, ArXiv e-prints, arXiv:0807.3614

Ansari, R., Campagne, J. E., Colom, P., et al. 2012, A\&A, 540, A129

Barkana, R., \& Loeb, A. 2007, RPPh, 70, 627

Bartolo, N., Komatsu, E., Matarrese, S., \& Riotto, A. 2004, Phys. Rep., 402, 103

Bernardeau, F., Colombi, S., Gaztañaga, E., \& Scoccimarro, R. 2002, Phys. Rep., 367, 1

Beutler, F., Saito, S., Seo, H.-J., et al. 2014, MNRAS, 443, 1065

Bharadwaj, S., \& Pandey, S. K. 2003, JApA, 24, 23

Blake, C., \& Glazebrook, K. 2003, ApJ, 594, 665

Booth, R. S., de Blok, W. J. G., Jonas, J. L., \& Fanaroff, B. 2009, ArXiv e-prints, arXiv:0910.2935

Camera, S., Santos, M. G., Ferreira, P. G., \& Ferramacho, L. 2013, Phys. Rev. Lett., 111, 171302

Chang, T.-C., Pen, U.-L., Bandura, K., \& Peterson, J. B. 2010, Nature, 466, 463

Chang, T.-C., Pen, U.-L., Peterson, J. B., \& McDonald, P. 2008, Phys. Rev. Lett., 100, 091303

Chen, X. 2010, AdAst, 2010, arXiv:1002.1416

—. 2011, SSPMA, 41, 1358

—. 2012, IJMPS, 12, 256

Cooray, A., \& Sheth, R. 2002, Phys. Rep., 372, 1

Creminelli, P. 2003, J. Cosmology Astropart. Phys., 10, 3

Dalal, N., Doré, O., Huterer, D., \& Shirokov, A. 2008, Phys. Rev. D, 77, 123514

D’Aloisio, A., Zhang, J., Jeong, D., \& Shapiro, P. R. 2013, MNRAS, 428, 2765

Desjacques, V., Jeong, D., \& Schmidt, F. 2011, Phys. Rev. D, 84, 063512

Fry, J. N. 1984, ApJ, 279, 499

Giannantonio, T., Ross, A. J., Percival, W. J., et al. 2014, Phys. Rev. D, 89, 023511

Giovanelli, R., Haynes, M. P., Kent, B. R., et al. 2005, AJ, 130, 2598

Gong, J.-O., \& Takahashi, T. 2014, Phys. Rev. D, 89, 023516

Gong, Y., Chen, X., Silva, M., Cooray, A., \& Santos, M. G. 2011, ApJ, 740, L20

Guzzo, L., Pierleoni, M., Meneux, B., et al. 2008, Nature, 451, 541 
Hall, A., Bonvin, C., \& Challinor, A. 2013, Phys. Rev. D, 87, 064026

Jeong, D., \& Komatsu, E. 2009, ApJ, 703, 1230

Johnston, S., Taylor, R., Bailes, M., et al. 2008, ExA, 22, 151

Liguori, M., Sefusatti, E., Fergusson, J. R., \& Shellard, E. P. S. 2010, AdAst, 2010, arXiv:1001.4707

Linder, E. V. 2008, APh, 29, 336

Loeb, A., \& Barkana, R. 2001, ARA\&A, 39, 19

Mao, Y., Tegmark, M., McQuinn, M., Zaldarriaga, M., \& Zahn, O. 2008, Phys. Rev. D, 78, 023529

Masui, K. W., Schmidt, F., Pen, U.-L., \& McDonald, P. 2010, Phys. Rev. D, 81, 062001

Masui, K. W., Switzer, E. R., Banavar, N., et al. 2013, ApJ, 763, L20

Matarrese, S., \& Verde, L. 2008, ApJ, 677, L77

McQuinn, M., Zahn, O., Zaldarriaga, M., Hernquist, L., \& Furlanetto, S. R. 2006, ApJ, 653, 815

Mo, H. J., Jing, Y. P., \& White, S. D. M. 1997, MNRAS, 284, 189

Morales, M. F. 2005, ApJ, 619, 678

Nan, R., Peng, B., Zhu, W., et al. 2000, in Astronomical Society of the Pacific Conference Series, Vol. 213, Bioastronomy 99, ed. G. Lemarchand \& K. Meech, 523

Parsons, A. R., Pober, J. C., Aguirre, J. E., et al. 2012, ApJ, 756, 165

Peacock, J. A. 1997, MNRAS, 284, 885

Peterson, J. B., Bandura, K., \& Pen, U. L. 2006, ArXiv Astrophysics e-prints, astro-ph/0606104

Peterson, J. B., Aleksan, R., Ansari, R., et al. 2009, in Astronomy, Vol. 2010, astro2010: The Astronomy and Astrophysics Decadal Survey, 234

Pillepich, A., Porciani, C., \& Matarrese, S. 2007, ApJ, 662, 1

Planck Collaboration, Ade, P. A. R., Aghanim, N., et al. 2013a, ArXiv e-prints, to appear in A\&A, arXiv:1303.5076

—. 2013b, ArXiv e-prints, arXiv:1303.5084

Rawlings, S., Abdalla, F. B., Bridle, S. L., et al. 2004, New A Rev., 48, 1013

Samushia, L., Reid, B. A., White, M., et al. 2014, MNRAS, 439, 3504

Scoccimarro, R., Colombi, S., Fry, J. N., et al. 1998, ApJ, 496, 586 
Scoccimarro, R., Sheth, R. K., Hui, L., \& Jain, B. 2001, ApJ, 546, 20

Sefusatti, E. 2009, Phys. Rev. D, 80, 123002

Sefusatti, E., \& Komatsu, E. 2007, Phys. Rev. D, 76, 083004

Seo, H.-J., Dodelson, S., Marriner, J., et al. 2010, ApJ, 721, 164

Seo, H.-J., \& Eisenstein, D. J. 2003, ApJ, 598, 720

Shaw, J. R., Sigurdson, K., Pen, U.-L., Stebbins, A., \& Sitwell, M. 2014, ApJ, 781, 57

Sheth, R. K., \& Tormen, G. 1999, MNRAS, 308, 119

Switzer, E. R., Masui, K. W., Bandura, K., et al. 2013, MNRAS, 434, L46

Tasinato, G., Byrnes, C. T., Nurmi, S., \& Wands, D. 2013, Phys. Rev. D, 87, 043512

Tegmark, M. 1997, Phys. Rev. Lett., 79, 3806

Thompson, A. R., Moran, J. M., \& Swenson, Jr., G. W. 2001, Interferometry and Synthesis in Radio Astronomy, 2nd Edition

Verde, L. 2010, AdAst, 2010, arXiv:1001.5217

Wang, X., Chen, X., Zheng, Z., et al. 2009, MNRAS, 394, 1775

Wang, Y. 2008, J. Cosmology Astropart. Phys., 5, 21 